\title{
Reduced-order Coupled Bidirectional Modeling of the Roll-N-Cage Isolator with Application to the Updated Bridge Benchmark
}

\author{
Giovanni De Mari ${ }^{1}$, Marco Domaneschi ${ }^{1 *}$, Mohammed Ismail ${ }^{2}$, Luca Martinelli ${ }^{1}$, \\ Josè Rodellar ${ }^{3}$ \\ ${ }^{1}$ Department of Civil and Environmental Engineering, Politecnico di Milano, \\ Milan, Italy \\ ${ }^{2}$ Structural Engineering Department, Zagazig University, Zagazig, Egypt \\ ${ }^{3}$ Department of Applied Mathematics III, Universitat Politècnica de Catalunya, \\ Barcelona, Spain
}

\begin{abstract}
This work presents and assesses a reduced model of a recently proposed seismic isolation system, namely the Roll-N-Cage one. This system incorporates several interesting features and mechanisms in a single unit; among the others: isolation, energy dissipation, buffering, high vertical stiffness and strength for supporting heavy loads. Departing from previous studies, which were based on the simulation of the Roll-N-Cage device by uncoupled orthogonal unidirectional models, this work first introduces a reduced order coupled bidirectional numerical model of the device. Then the efficiency of such type of control devices, as simulated by the proposed approach, is evaluated for the seismic protection of a cable supported bridge. The bridge model is derived from the ASCE benchmark cable-stayed bridge by means of an extended version.
\end{abstract}

Keywords: Laboratory, Model, Simulation, Isolation, Cable-stayed, Earthquake.

${ }^{(*)}$ Corresponding author: $\mathrm{T}+39.02 .2399 .4267 ; \mathrm{F}+39.02 .2399 .4220 ; \mathrm{E}$ marco.domaneschi@polimi.it; Department of Civil and Environmental Engineering, Politecnico di Milano, P.zza Leonardo da Vinci 32, 20133-Milano I. 


\section{Introduction}

A new seismic isolation system, namely the Roll-N-Cage one (RNC in the following), has been recently [1] proposed in the literature. This system incorporates several interesting features and mechanisms in a single unit; among the others: isolation, energy dissipation, buffering. It also can provide stiffness against low loading levels (e.g. wind and traffic), a great range of horizontal flexibility, high vertical stiffness and strength for supporting heavy dead loads. Re-centering and no uplift during its lateral motion are also interesting features which can be implemented alternatively. As a completion of a previous studies [2,3] on the simulation of the Roll-N-Cage device by uncoupled orthogonal unidirectional models, this work first introduces a reduced order coupled bidirectional numerical model of the device. Then the efficiency of such type of control devices, as simulated by the proposed approach, is evaluated for the seismic protection of cable supported bridges.

The case study herein selected is the updated ASCE benchmark model on cable-stayed bridges [4]. The refined bridge model is developed in the ANSYS [5] multipurpose finite element environment so as to include relevant modeling aspects, in the simulation of the stay cables dynamics, in the implementation of the seismic external excitation, in the soil-structure interaction and to compare control strategies for the mitigation of the seismic effects.

The RNC devices are introduced into the finite element numerical model by an external userelement, implemented in an independent executable program called by the main script that controls the analysis in ANSYS. The numerical problem is then solved with a mixed implicitexplicit approach in the time domain through the finite element code solver (Newmark) and, externally to this code, the solution of the differential bidirectional model (Runge-Kutta).

Analyses are carried out in the time domain for a multiple support seismic excitation. The vertical component of the earthquake is considered as well. The seismic input, which consequently is not the same on all the supports, satisfies a fixed coherence function. The soil type regulates the degree of correlation.

\section{The RNC Device}

The Roll-N-Cage (RNC) isolator has been recently proposed $[1,6]$ as an attempt to enhance aseismic design. It is a rolling-based isolation system that allows to achieve the maximum possible structure-ground decoupling and, therefore, to minimize the seismic force transfer to the isolated structure. It provides in a single unit all the necessary functions of vertical rigid support, horizontal flexibility with enhanced stability, hysteretic energy dissipation and resistance to minor vibration loads. Three unique features distinguish the RNC isolator: 1) a self-stopping (buffer) mechanism to limit the isolator displacement under severe seismic excitations to a predetermined value; 2) a linear gravity-based self-recentering mechanism that prevents residual dislocations after earthquakes; and 3) a remarkable ability to resist vertical tension by means of its metallic yield dampers. To obtain the most benefit of the adopted rolling-based motion mechanism, which requires less lateral forces to initiate and maintain high degree of structure-ground decoupling compared to other motion mechanisms of the elastomeric-based and friction-based isolation systems, the RNC isolator is provided with a lateral pre-yield stiffness mechanism, against minor vibration loads, independent to its bearing mechanism. This independency allows for accurate tuning of the initial pre-yield stiffness to permit the commencement of the seismic isolation process, or structure-ground decoupling, just after the seismic forces exceed the maximum limit of minor vibration loads, contrary to the available isolation systems. The RNC isolator can be available in different forms to suit the structure or object to be protected regarding mass, size, uni or multidirectional isolation and the maximum allowed seismic gaps between adjacent structures, as shown in Figure 1(a, b, c). The RNC isolator was numerically characterized in [2], mathematically modeled in [7], and experimentally investigated in [8] using [9]. Figure $1(d, e)$ shows a $1 / 10$ reduced scale experimental prototypes, of the design form adequate for light to moderate mass structures, at neutral and maximum deformed positions, respectively. 

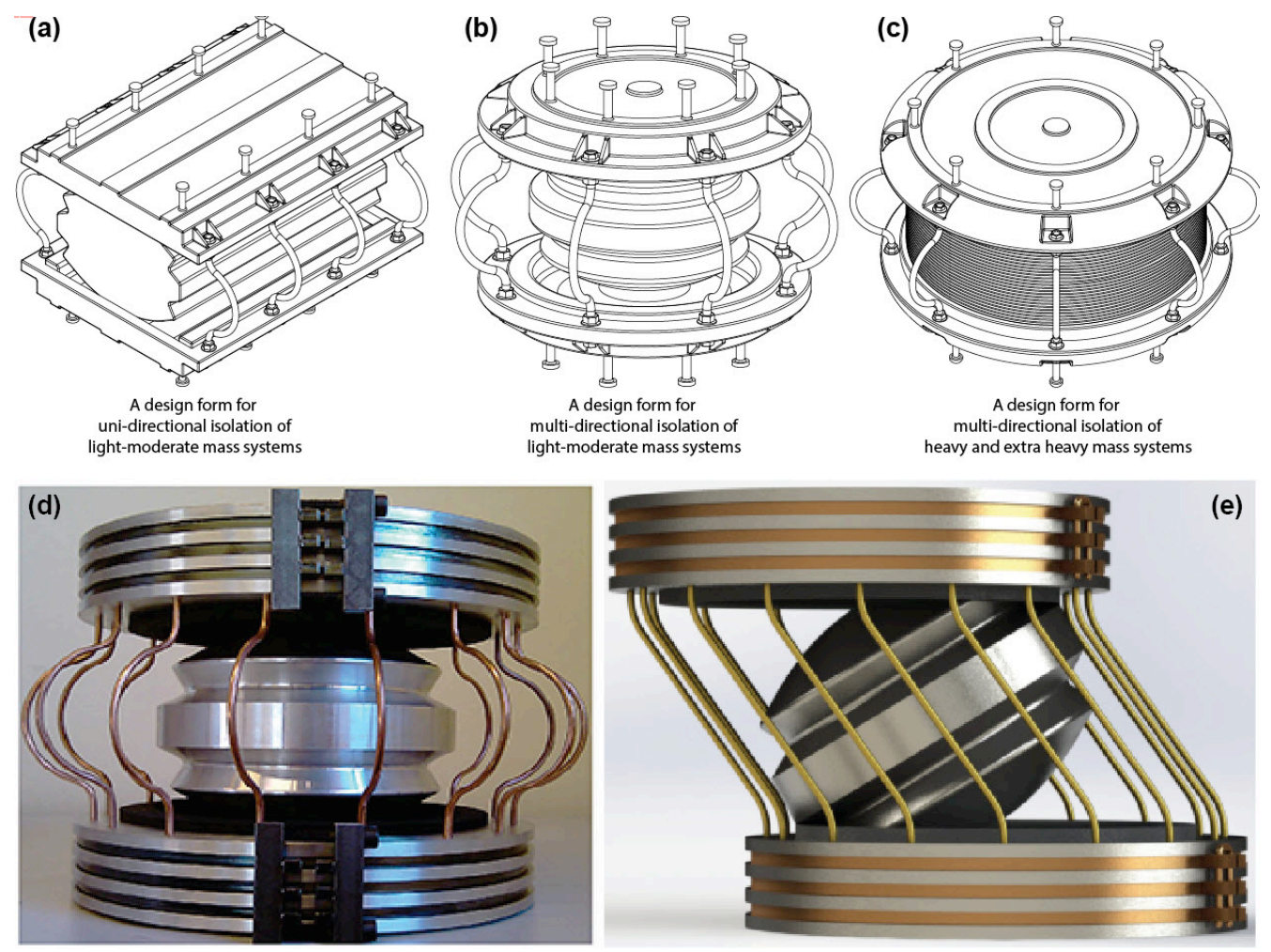

Figure 1: the RNC isolator: (a) a design form for uni-directional isolation of light-moderate mass systems; (b) a design form for multi-directional isolation of light-moderate mass systems; (c) a design form for multi-directional isolation of heavy and extra heavy mass systems; (d) a smallscale physical prototype at neutral position; (e) a small-scale prototype at maximum deformed position.

\section{LABORATORY TESTS ON THE PHYSICAL PROTOTYPE: A NOVEL TESTING PLATFORM}

A novel multi-purpose mechanical extension is designed for a Stewart Platform (Hexapod), and later patented [9], to enable the Hexapod (shown in Figure 2a) to perform up to fifteen standard mechanical tests, besides maintaining its main function as a motion simulator. Figures $2 \mathrm{~b}-2 \mathrm{~d}$ demonstrate one part of the designed mechanical extension. This part is suitable for testing experimental block-like, cylindrical and seismic isolation bearing specimens under tension, compression, shear, torsion and fatigue tests. Figure 3a shows a real constructed example of the designed mechanical extension after being integrated to the Hexapod. Some sample real photos of a RNC isolator experimental prototype under cyclic horizontal shear displacement are shown in Figures 3b-3d at neutral and different deformed positions. More details about the designed mechanical extension for Hexapod are found in [9].

An extensive series of numerical simulation tests were carried out in [2] to identify the mechanical characteristics of the RNC isolator using real-scale and small-scale prototypes numerically. These tests have been followed by thorough experimental verification using several sets of the 1/10 small-scale prototypes only of the RNC isolator having different configurations, characteristics and materials in [10]. Two samples of the used experimental prototypes are shown in Figures 1d-1e; one is made of stiff-aluminum (with a design compressive capacity of $5 \mathrm{kN}$ ) as shown in Figure $1 \mathrm{~d}$, and the other one is made of Delrin (with a design compressive capacity of $3 \mathrm{kN}$ ) as shown in Figure 1e.

The lower plate of a RNC isolator prototype is tightly attached to the lower fixed base of the developed testing platform, while the upper plate of the prototype is fastened to the upper 
moving base of the developed testing platform, see Figs 2 and 3. Synthetic sinusoidal and reduced-amplitude real seismic input shear displacements are applied separately to the upper moving base to generate a relative shear motion within the RNC isolator prototypes (between its top moving part and the lower fixed one). The experimental prototype responds to such relative shear displacement through rolling of the inner core and extension of the metallic yield dampers, arranged around the rolling core, as demonstrated by Figures $3 \mathrm{c}-3 \mathrm{~d}$. The input displacement is measured by a laser sensor to confirm that there are no losses, while the output shear force is measured by a precise force/moment sensor, which is located as shown in Figure 2c. Two amplitudes of a chosen input shear displacement are applied to avoid activation (unscaled) and activate (scaled) the inherent buffer mechanism of the RNC isolator. To represent the results in a real sense, the reduced-scale input-output data of the reducedscale experimental prototypes are transformed into the real scale (before being reduced) using dimensional analysis. Two real-scale sample input-output data are plotted in Figs 2 and 3 using synthetic sinusoidal and real seismic ground motions, respectively, and considering scaled and unscaled amplitudes to activate and deactivate the buffer mechanism, respectively.

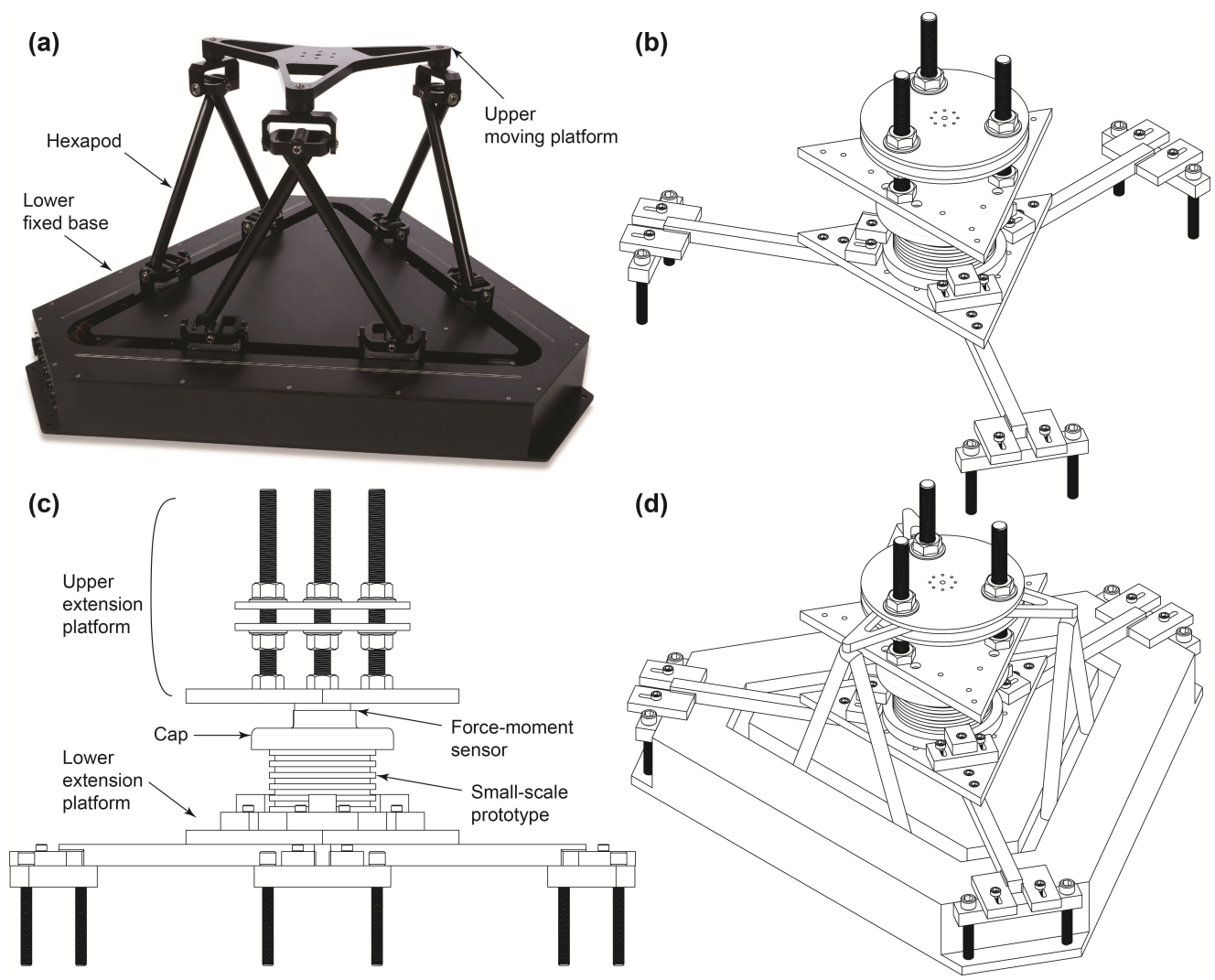

Figure 2: mechanical Hexapod extension: (a) Hexapod; (b) 3D view of the designed Hexapod extension; (c) elevation view of the designed Hexapod extension; (d) the designed extension incorporated to Hexapod. 


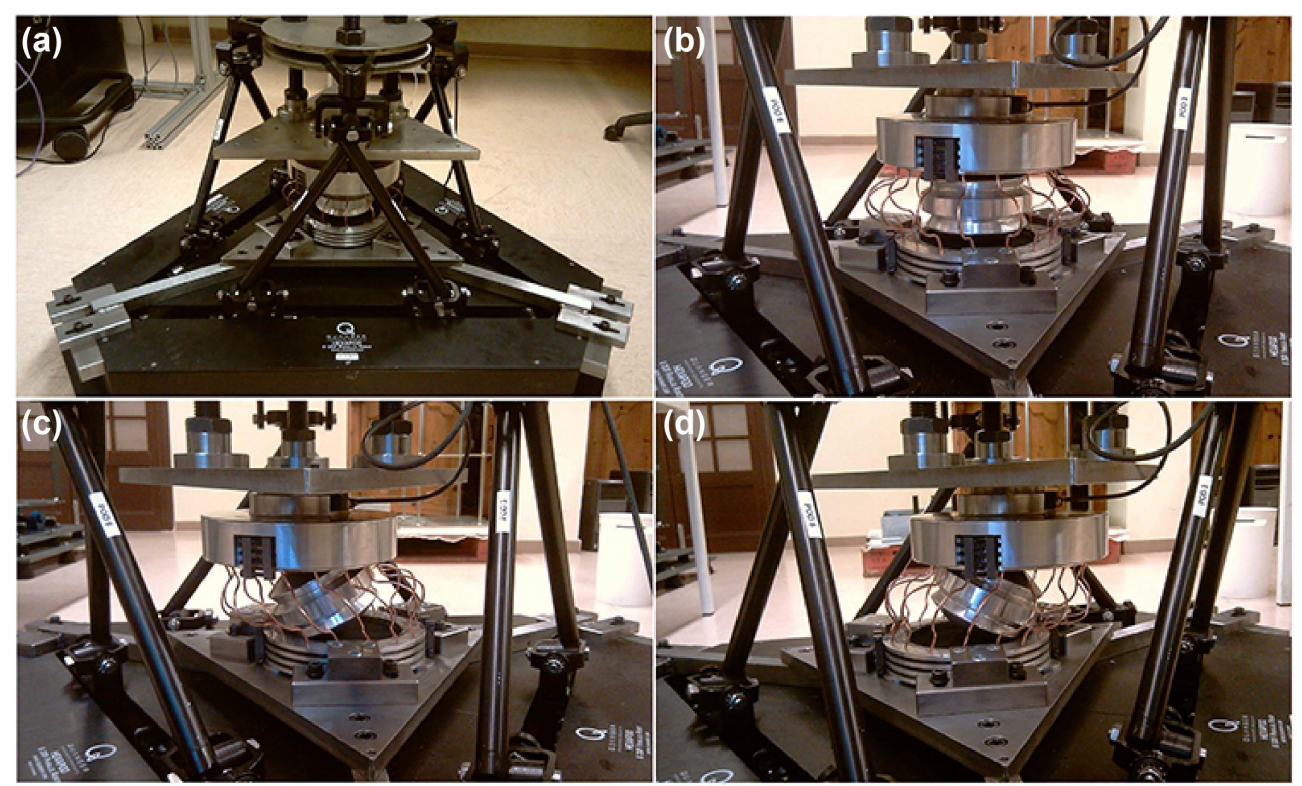

Figure 3: (a) mechanical extension incorporated to Hexapod; (b) neutral position; (c) to-the-left deformed position; (d) to-the-right deformed position.

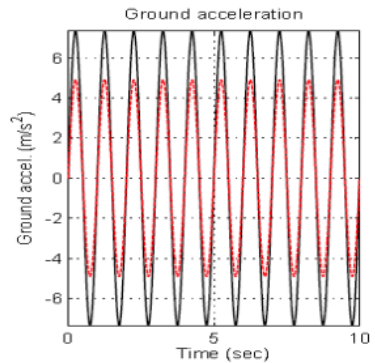

a)

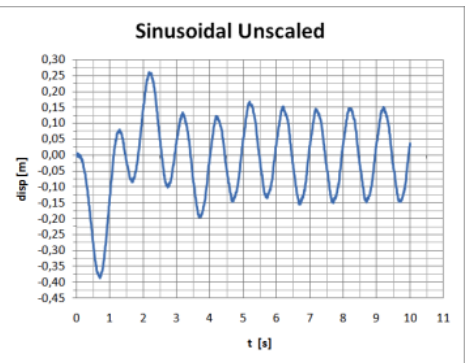

b)

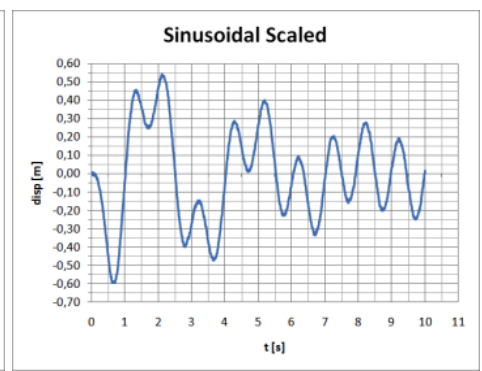

c)

Figure 4: sinusoidal test. (a) Unscaled and Scaled inputs. (b), (c) Device outputs.

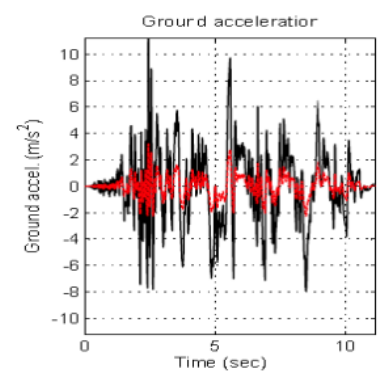

a)

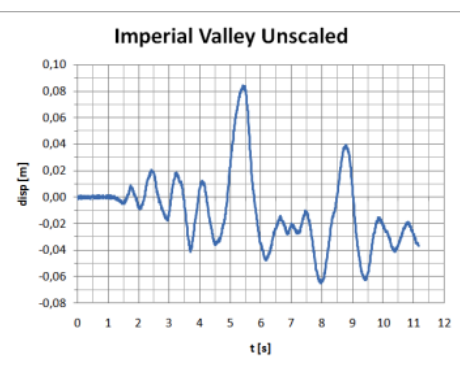

b)

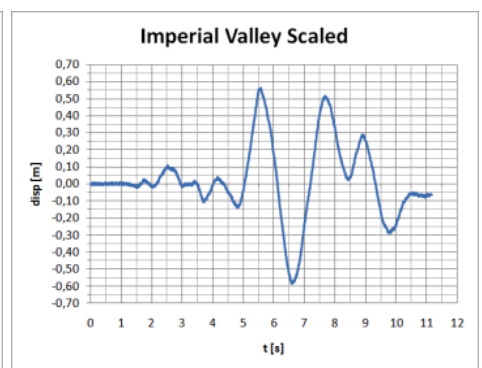

c)

Figure 5: seismic test. (a) Unscaled and Scaled inputs (Imperial Valley). (b), (c) Device outputs.

Figure 3 anticipates the performed laboratory tests on the RNC device prototype. The monitoring system on the Hexapod allows to collect input and output quantities as reported in the following Figures 4 and 5. They depict respectively the results from the sinusoidal and seismic 
unidirectional tests on the physical 1/10th small-scale prototype in laboratory, which has a circular shape (see Figure 1b) with 16 metallic bars (diameter $3 \mathrm{~mm}$ ) uniformly distributed with angular distance of $22.5^{\circ}$ on the circumference of a circle of $188 \mathrm{~mm}$ in diameter. The metallic bars and the rolling body are in mild-steel with elastic modulus of $210000 \mathrm{MPa}$, Poisson coefficient 0.3 , yielding stress $240 \mathrm{MPa}$, post-yielding modulus $2 \%$ of the elastic one. The geometry and the dimensions are also detailed in Figure 2 of reference [2].

The outcomes from Figure 4 and 5 will be useful for the modeling developments and parameters identification which will be presented in the following numerical improvements.

\section{REDUCED MODEL OF RNC DEVICES}

The reduced model of RNC devices introduced in this work adopts the coupled bi-directional formulation [12] initially proposed, in a different framework, by Park et al. as an expansion of the Bouc-Wen [13] mono-directional hysteretic model. The Park et al. model can be seen, as it can the Bouc-Wen one, as a black-box semi-physical model which can match an hysteretic behavior of a device by properly tuning its parameters.

In this work a modified model is used to characterize the RNC isolator device with its distinctive buffer mechanism. An independent executable program simulate the isolation devices in the structural analyses performed with the finite element commercial code ANSYS [5]. This program is to be called by the main script controlling the ANSYS analysis, and is interfaced with the ANSYS database for the analysis through interface routines written in APDL (ANSYS Parametric Design Language). In particular, the process of calling the external executable from the ANSYS script is carried out using the APDL command "/SYS" [11].

\section{A. Bi-directional hysteresis: basic formulations}

Following the formulation in [12], the restoring force vector in a hysteretic system may be expressed as:

$$
\left\{\begin{array}{l}
q_{x} \\
q_{y}
\end{array}\right\}=\alpha[\boldsymbol{K}]\left\{\begin{array}{l}
u_{x} \\
u_{y}
\end{array}\right\}+(1-\alpha)[\boldsymbol{K}]\left\{\begin{array}{l}
Z_{x} \\
Z_{y}
\end{array}\right\}
$$

in which $u$ is the displacement vector with orthogonal components $u_{x}$ and $u_{y}$, in $x$ and $y$ directions respectively; $q$ the restoring force vector with components $q_{x}$ and $q_{y}$; $\boldsymbol{K}$ is the initial stiffness matrix; $\alpha$ is the post-yielding stiffness ratio; $Z$ represents the hysteretic displacement vector.

For isotropic hysteretic restoring forces, $Z_{x}$ and $Z_{y}$ satisfy the following coupled differential equations:

$$
\begin{aligned}
& \dot{Z}_{x}=A \dot{u}_{x}-\beta\left|\dot{u}_{x} Z_{x}\right| Z_{x}-\gamma \dot{u}_{x} Z_{x}^{2}-\beta\left|\dot{u}_{y} Z_{y}\right| Z_{x}-\gamma \dot{u}_{y} Z_{x} Z_{y} \\
& \dot{Z}_{y}=A \dot{u}_{y}-\beta\left|\dot{u}_{y} Z_{y}\right| Z_{y}-\gamma \dot{u}_{y} Z_{y}^{2}-\beta\left|\dot{u}_{x} Z_{x}\right| Z_{y}-\gamma \dot{u}_{x} Z_{y} Z_{x}
\end{aligned}
$$

where $A, \beta, \gamma$ are non-dimensional parameters that control the shape and size of the hysteresis cycle.

\section{B. Mathematical modeling of the buffer mechanism}

The RNC device has a peculiar built-in buffer mechanism that needs to be modeled along with its hysteretic behavior to fully characterize the isolator device. 


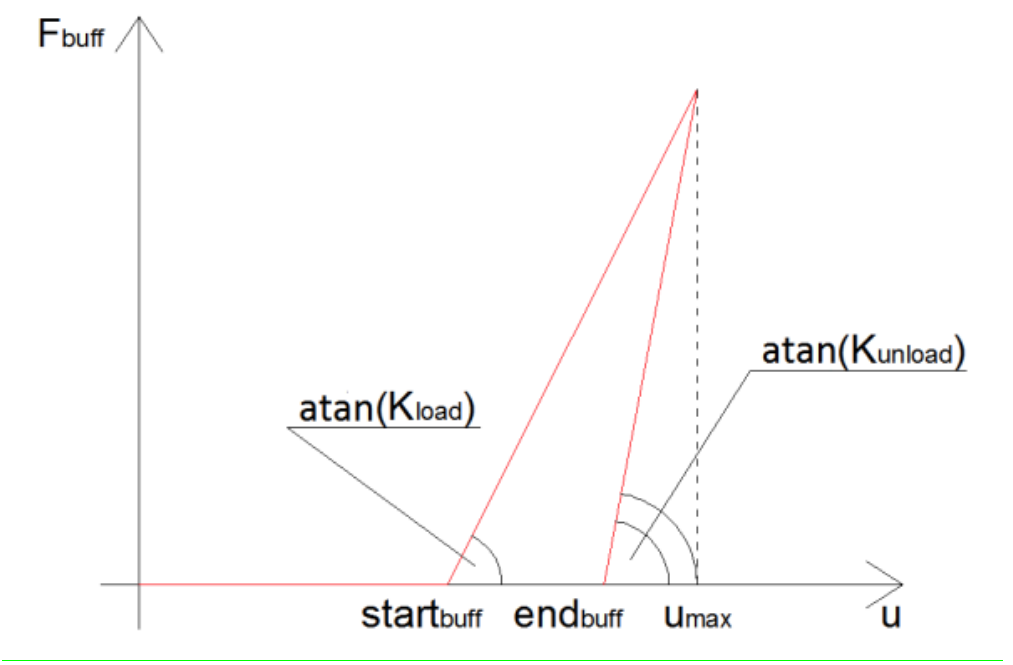

Figure 6: model of the buffering mechanism in the RNC device where "atan" stands for the inverse of the tangent trigonometric function.

Guided from physical experimentation of a prototype device [8], the amount of the total restoring force due to the buffer mechanism, may be expressed as (see Figure 6):

$$
\begin{gathered}
F_{\text {buff }, x}=\left\{\begin{aligned}
0, & u_{x} \leq \text { start_buff } \\
K_{\text {load }}\left(u_{x}-\text { start_buff }\right), & u_{x}>\text { start_buff } \text { and } \dot{u}_{x}>0 \\
K_{\text {unload }}\left(u_{x}-\text { end_buff } f_{x}\right), & u_{x}>\text { end_buff } f_{x} \text { and } \dot{u}_{x}<0
\end{aligned}\right. \\
F_{\text {buff }, y}=\left\{\begin{aligned}
0, & u_{y} \leq \text { start_buff } \\
K_{\text {load }}\left(u_{y}-\text { start_buff }\right), & u_{y}>\text { start_buff } \text { and } \dot{u}_{y}>0 \\
K_{\text {unload }}\left(u_{y}-\text { end_buff } f_{y}\right), & u_{y}>\text { end_buff } f_{y} \text { and } \dot{u}_{y}<0
\end{aligned}\right.
\end{gathered}
$$

Where $F_{\text {buff, } x}$ e $F_{\text {buff,y }}$ are the buffer restoring forces in $x$ - and $y$-direction respectively, $K_{\text {load }}$ and $K_{\text {unload }}$ are the loading and unloading stiffness, start_buff is the displacement (device feature) which lets the buffer mechanism start (when the rolling body and the less stiff plate are just in touch), while end_buff $x$ and end_buff $y$ are the displacements, valued imposing the buffer force in the unloading path equal to zero, for which the buffer effect stops and the restoring force returns to be just the hysteretic one. The end buffer displacements depend not only on both stiffness and start_buffer displacement, but also on the maximum (minimum) displacement reached in the loading path, so they may be expressed as:

$$
\begin{aligned}
& \text { end_buff } f_{x}=\frac{\left(K_{\text {unload }} u_{\max , x}-K_{\text {load }}\left(u_{\max , x}-s_{\text {unload }}\right.\right.}{\left.\left.K_{\text {urtbuff }}\right)\right)} \\
& \text { end_buff } f_{y}=\frac{\left(K_{\text {unload }} u_{\text {max }, y}-K_{\text {load }}\left(u_{\text {max }, y}-\text { start_buff }\right)\right)}{K_{\text {unload }}}
\end{aligned}
$$

The bidirectional formulation of the buffer mechanism can be easily applied to monodirectional loading conditions giving great accordance with the results of mono-directional physical tests as is demonstrated in the following subsection. 


\section{Parameters identification}

Two different identification procedures are performed in this work: one based on experimental results, coming from lab tests made for the very first time on a physical small-scale prototype (1/10th) of the RNC isolator (Figures 4 and 5 in particular), one based on numerical simulations carried out on a FEM model of the device. A constrained nonlinear least square optimization algorithm, available in MATLAB [14], is used to obtain the model parameters in both procedures.

\section{Optimization algorithm}

The least square curve fitting function lsqnonlin is chosen from the ones available in the MATLAB Optimization toolbox. As reported in the program guide, lsqnonlin solves nonlinear least-squares problems, including nonlinear data-fitting problems. Rather than compute the sum of squares, $\|f(x)\|_{2}^{2}$, lsqnonlin requires the user-defined function to compute the vector-valued function:

$$
f(x)=\left[\begin{array}{c}
f_{1}(x) \\
f_{2}(x) \\
\vdots \\
f_{n}(x)
\end{array}\right]
$$

Then, in vector terms, this optimization problem can be restated as:

$$
\min _{x}\|f(x)\|_{2}^{2}=\min _{x}\left(f_{1}(x)^{2}+f_{2}(x)^{2}+\cdots+f_{n}(x)^{2}\right)
$$

Where $x$ is a vector and $f(x)$ is a function that returns a vector value. The following syntax is used for the function:

$$
x=\text { lsqnonlin }(\text { fun }, x 0, l b, u b)
$$

where:

- $\quad x$ represents the parameters set to identify;

- $\quad f u n$ is the function to minimize: it should return a vector of values and not the sum of squares of the values, because the algorithm implicitly sums and squares $\operatorname{fun}(x)$;

- $x_{0}$ is the vector of initial parameters values, that represents the point in which the procedure starts;

- $\quad l_{b}$ and $u_{b}$ define a set of lower and upper bounds on the design variables in $x$, so that the solution is always in the range $l_{b} \leq x \leq u_{b}$.

Within this study, the function to minimize is represented by the difference between the device restoring force vector, given by the test results, $q_{\text {test }}$, and those of the proposed mathematical model, $\mathrm{q}_{\text {math }}$, with their two in-plane components, $f_{1}=\left(q_{\text {test }, x}-q_{\text {math }, x}\right)$ and $f_{2}=\left(q_{\text {test }, y}\right.$ $\left.q_{\text {math,y }}\right)$. It can also be summarized as it follows:

$f=\left[\begin{array}{l}f_{1} \\ f_{2}\end{array}\right]=\left(q_{\text {test }}-q_{\text {math }}\right)=\left[\begin{array}{l}q_{\text {test }, x}-q_{\text {math }, x} \\ q_{\text {test }, y}-q_{\text {math }, y}\end{array}\right]$

The vectors defining the lower and upper bounds of the parameters to identify have been set taking into account what is reported in $[12,13]$.

Other algorithm options used are the following: the maximum number of iterations allowed (MaxIter), a positive integer, is set to 400 while the termination tolerance on the function value (TolFun) and the termination tolerance on $x$ (TolX), positive scalars, are both set to 1E-6.

The number of iterations required for the identification procedures varies from a minimum of 13 (for the buffer mechanism parameters) to a maximum of 27 (for the Park et al. model parameters). In all cases the procedure stopped because of the TolFun criterion. 


\section{Identification from laboratory tests}

The identification of the Park et al. model parameters to reproduce the RNC behaviour was based on experimental results [8] coming from lab tests made for the very first time on a physical small-scale prototype of the RNC isolator at the Control, Dynamics and Applications Laboratory (CoDAlab), UPC, Barcelona. The tests were made, using dimensional analysis, on a small-scale (1/10th) prototype of a real-scale isolator (see Figures 2, 3). Two different acceleration histories were given as input to the physical prototype: the sinusoidal one in Figure 4 and the one recorded during the Imperial Valley earthquake, in Figure 5. The input were afterward scaled to activate the built-in buffer mechanism of the RNC device.
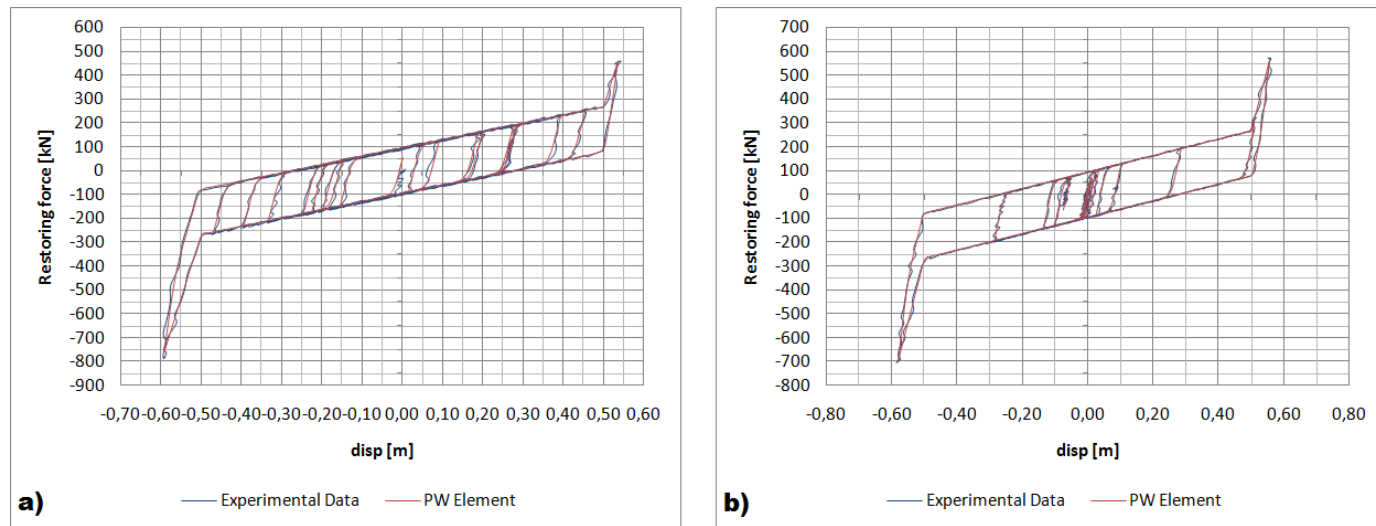

Figure 7: match between Park et al. model with the identified paramenters in Table I and the experimental data from the physical test of the RNC device when buffering mechanism is activated: (a) sinusoidal input; (b) Imperial Valley input.

The Park et al. model parameters $(A, \beta, \gamma, \alpha, K)$ and the buffer mechanism parameters $\left(K_{\text {load }}\right.$, $\left.K_{\text {unload }}\right)$ identified form the experimental data with the procedure described in section C) are listed in Table I, while Figure 7 shows the match between the Park et al. model (using the parameters in Table I) and the experimental outcome from the testing of the physical prototype in the case of activation of the buffer mechanism.

It's worth noting that the match is satisfying for both the sinusoidal and the Imperial Valley input, even though only the sinusoidal one was used for the identification procedure, and consequently the Imperial Valley serves the purpose of a blind test.

\begin{tabular}{|l|l|}
\hline Parameter of Park et al. model & \multicolumn{1}{|c|}{ Value } \\
\hline$A$ & 0.8081 \\
\hline$K$ & $7920.1 \mathrm{kN} / \mathrm{m}$ \\
\hline$\alpha$ & 0.0435 \\
\hline$\beta$ & 2592.1 \\
\hline$\gamma$ & 2591.1 \\
\hline start_buff & $50 \mathrm{~cm}$ \\
\hline$K_{\text {load }}$ & $5003.9 \mathrm{kN} / \mathrm{m}$ \\
\hline$K_{\text {unload }}$ & $5215.4 \mathrm{kN} / \mathrm{m}$ \\
\hline
\end{tabular}

Table 1: Parameters of Park et al. model

\section{Finite element reproduction of bidirectional loading paths}

Testing of isolation devices is traditionally uni-axial because of the complexity of loading in two directions. However, the earthquake response of an isolated bridge deck is two-dimensional, hence bi-directional characterization of the devices is crucial in order to understand the behavior 
and evaluate the efficacy of the devices and of their numerical models. In light of this observations, this section is devoted to deepen this aspect for the RNC isolator device.

The only components of the RNC device which exhibit a dissipative hysteretic behavior are the metallic bars surrounding the quasi-elliptical rotating body [2]. Depending on the initial configuration (initial curvature) and the displacement histories, they can lead to a peculiar response that should be avoided at the design phase. Should this not be feasible, we will in the following provide a method to include it into the Park et al. model.

To evaluate the response of the RNC to bidirectional excitations in the horizontal plane a 3D finite element model of the dissipative bars system has been first developed in the commercially available finite element ANSYS program [2,5] by consolidated numerical procedures such as an isotropic elastic-plastic material and kinematic bidirectional hardening for the mild-steel (elastic modulus $210000 \mathrm{MPa}$, Poisson coefficient 0.3 , yielding strength $240 \mathrm{MPa}$, post_yielding modulus $2 \%$ ), isoparametric 8-nodes brick elements, geometric nonlinearities.

To assess the effects (if any) of mesh dependency, different finite element sizes and element types have been used. The presence of the rolling body, and the related contact problems between surfaces, has been disregarded. Rotations of the top surface can be neglected and the distance between the top and bottom surface of the system is constant. The degrees of freedom for the nodes of the damping bars are restrained with a body constrain to the master node, while the base of each bar has been completely restrained. The kinematic boundary conditions imply that the master node only translate in a horizontal plane.

Different finite element sizes and element types used to asses the eventual presence of mesh dependency, triggered by non linear behavior of materials, make reference: the first to Timoshenko beam elements (ANSYS beam188 element [5]); the others to hexahedral solid elements (ANSYS solid45 element [5]). A reasonable equivalence in the results have been reached and the model depicted in Figure 8a has been considered the reference one. Figure 8b depicts the detail of the slave nodes at the top of a metallic bar. 


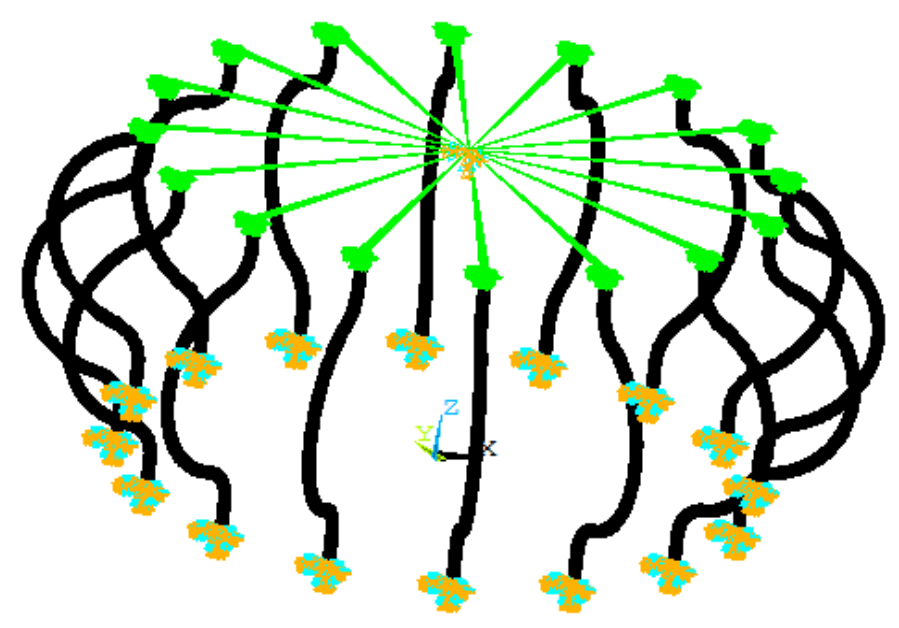

a)
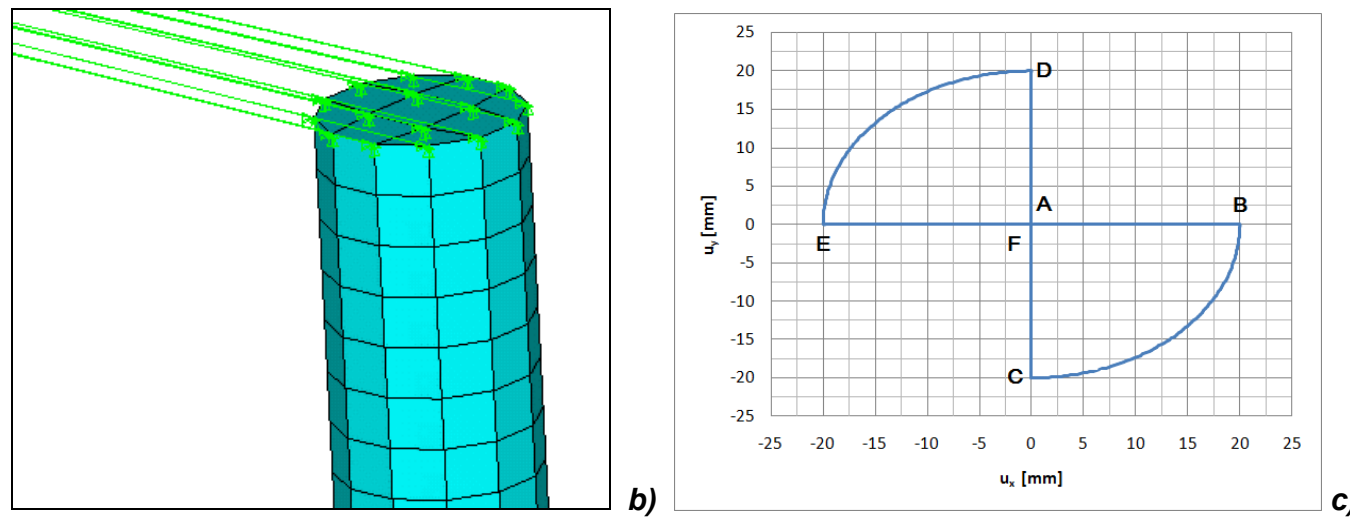

Figure 8: consolidated FE model (a), slave nodes at the top of a metallic bar (b), displacement path (c).

The finite element model has been subjected to several kinematic histories to obtain displacements-forces curves. In turn, these curves have been assumed as the target behaviors to be reproduced through the Park et al. law for further implementation on the bridge structural system [2]. First, the bidirectional displacement path shown in Figure 8c was applied to the finite element model, the displacements' history is assigned to the master node positioned in the centre of the bars system. The first branches of the displacement paths are uni-directional to allow for calibrating the parameters of a Bouc-Wen model to be used for uni-axial hysteretic cycles and in a simplified solution prior adopted. In this, two Bouc-Wen unidirectional models are employed together in an orthogonal-uncoupled scheme, as in [2]. The results from the bi-directional analysis coming from the finite element ANSYS program are compared to the outcomes from the bidirectional model developed from the Park et al. model, and to those from the simplified solution based on two Bouc-Wen unidirectional models, in Figure 9. This figure reports the comparison at a selected low level of displacements. Similar outcomes can been observed from both solutions with respect to the target, represented by the finite element model output. 

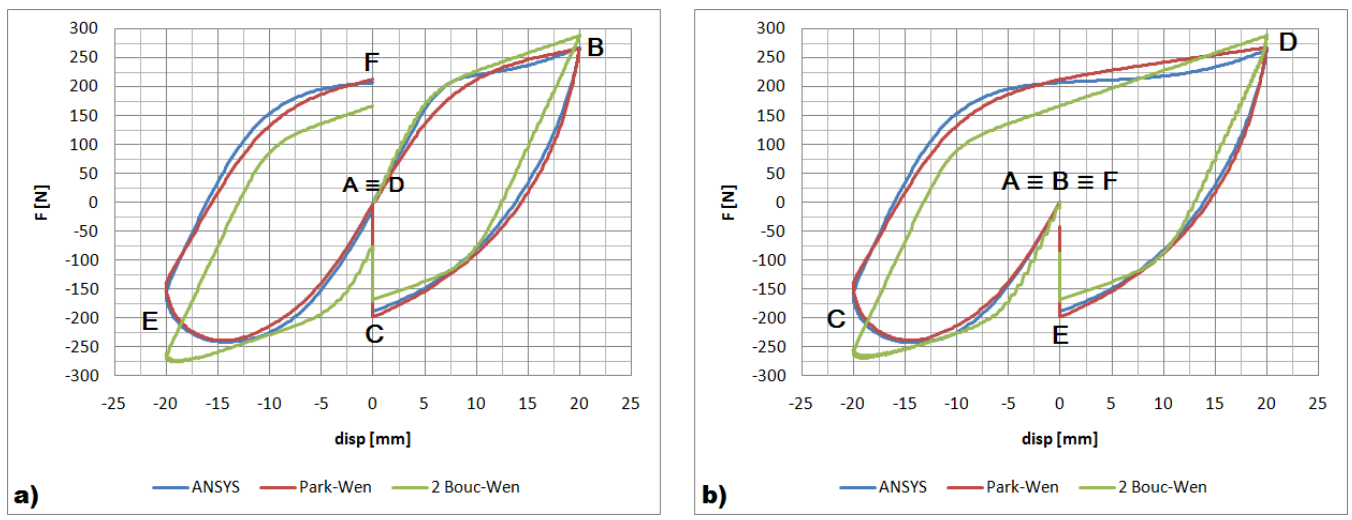

Figure 9: match between Park et al. model, ANSYS results and the device characterization previously adopted by the research group based on two independent Bouc-Wen models: (a) Xdirection; (b) y-direction
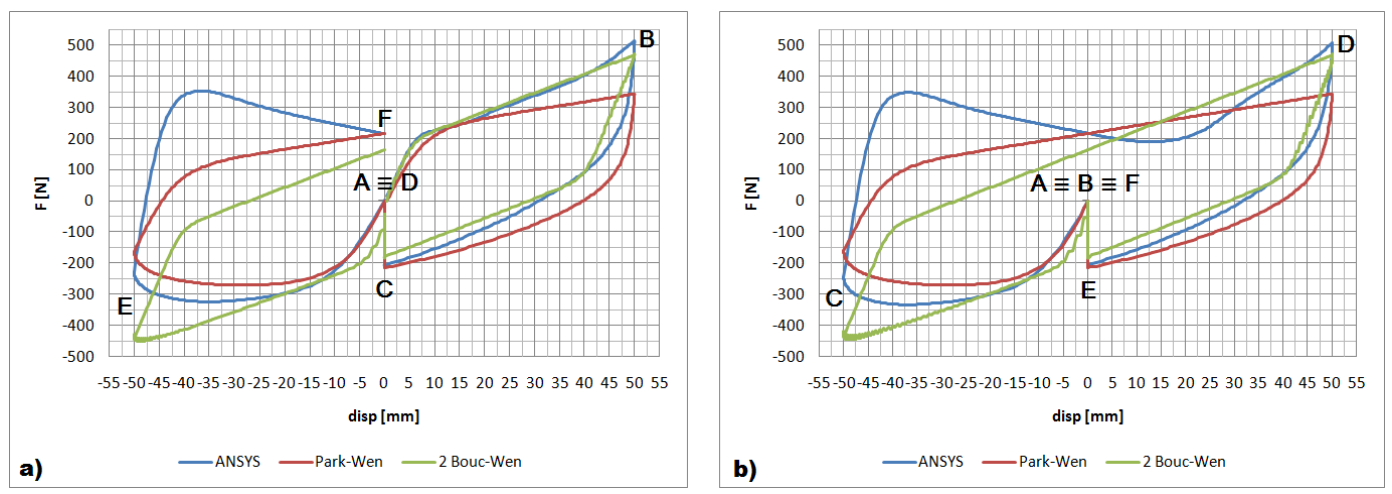

Figure 10: match for the scaled load path between Park et al. model, ANSYS results and the device characterization based on two independent Bouc-Wen models: (a) $x$-direction; (b) $y$-direction.

Figure 10 shows a similar comparison, but for larger imposed displacements obtained from having scaled the displacement path in input. From this picture, one senses that a Park et al. model, set-up with constant parameters, is not able to match some peculiar features of the device behavior related to the buckling of the metallic dampers. Buckling that occurs in the unloading branch, after having reached the tensile plastic range during the loading branch (see the limit conditions in Figures 3c-3d). To overcome this deficiency, in the following, a modification of the Park et. al. model is presented based on taking into account the separate variation of the parameter $\alpha$ in the equations of the system (1). Therefore, this variation is expressed in both $x$ and $y$-directions (with $i$ $=x, y$ and $j=y, x$ ), during the loading steps for the generic time instant $t_{k}$, as:

$$
\alpha_{i}\left(t_{k}\right)=\left\{\begin{aligned}
\alpha, & u_{i}\left(t_{k}\right)<u_{h} \\
\alpha+C\left(u_{i}\left(t_{k}\right)-u_{h}\right), & u_{i}\left(t_{k}\right) \geq u_{h} \text { and } u_{i}\left(t_{k-1}\right) \leq u_{h} \text { and } u_{j}\left(t_{k}\right)=0 \\
\alpha_{i}\left(t_{k-1}\right)+C\left(u_{i}\left(t_{k}\right)-u_{i}\left(t_{k-1}\right)\right), & u_{i}\left(t_{k}\right) \geq u_{h} \text { and } u_{i}\left(t_{k-1}\right)>u_{h} \text { and } u_{j}\left(t_{k}\right)=0 \\
\alpha+C\left(u_{i}\left(t_{k}\right)-u_{h}\right) / 2, & u_{i}\left(t_{k}\right) \geq u_{h} \text { and } u_{i}\left(t_{k-1}\right) \leq u_{h} \text { and } u_{j}\left(t_{k}\right) \neq 0 \\
\alpha_{i}\left(t_{k-1}\right)+C\left(u_{i}\left(t_{k}\right)-u_{i}\left(t_{k-1}\right)\right) / 2, & u_{i}\left(t_{k}\right) \geq u_{h} \text { and } u_{i}\left(t_{k-1}\right)>u_{h} \text { and } u_{j}\left(t_{k}\right) \neq 0
\end{aligned}\right.
$$

While, for the unloading steps it becomes: 
$\alpha_{i}\left(t_{k}\right)=\left\{\begin{aligned} \alpha, & u_{i}\left(t_{k}\right)<u_{h} \text { and }\left(u_{j}\left(t_{k}\right) \neq 0 \text { or } u_{\max , i}<u_{b}\right) \\ \bar{\alpha}_{i}-\left(\bar{\alpha}_{i}-\alpha\right) \frac{\left(u_{i}\left(t_{k}\right)-u_{\max , i}\right)}{\left(u_{h}-u_{\max , i}\right)}, & u_{i}\left(t_{k}\right) \geq u_{h} \text { and }\left(u_{j}\left(t_{k}\right) \neq 0 \text { or } u_{\max , i}<u_{b}\right) \\ -\alpha, & u_{i}\left(t_{k}\right)<u_{b} \text { and }\left(u_{j}\left(t_{k}\right)=0 \text { and } u_{\max , i}>u_{b}\right) \\ \bar{\alpha}_{i}-\left(\bar{\alpha}_{i}+\alpha\right) \frac{\left(u_{\max , i}-u_{i}\left(t_{k}\right)\right)}{\left(u_{\max , i}-u_{b}\right)}, & u_{i}\left(t_{k}\right) \geq u_{b} \text { and }\left(u_{j}\left(t_{k}\right)=0 \text { and } u_{\max , i}>u_{b}\right)\end{aligned}\right.$

Where $u_{h}$ is the displacement at which the hardening behavior commence due to a more straight configuration of the metallic dampers, $u_{b}$ is the displacement which takes into account the buckling effects above mentioned, $C$ is a constant that regulates the linear variation of alpha, while $\bar{\alpha}$ is the maximum value of alpha reached in during the loading path.

Figure 11 depicts the results obtained with this model refinement that allows to match correctly the device response also at larger displacements limit.
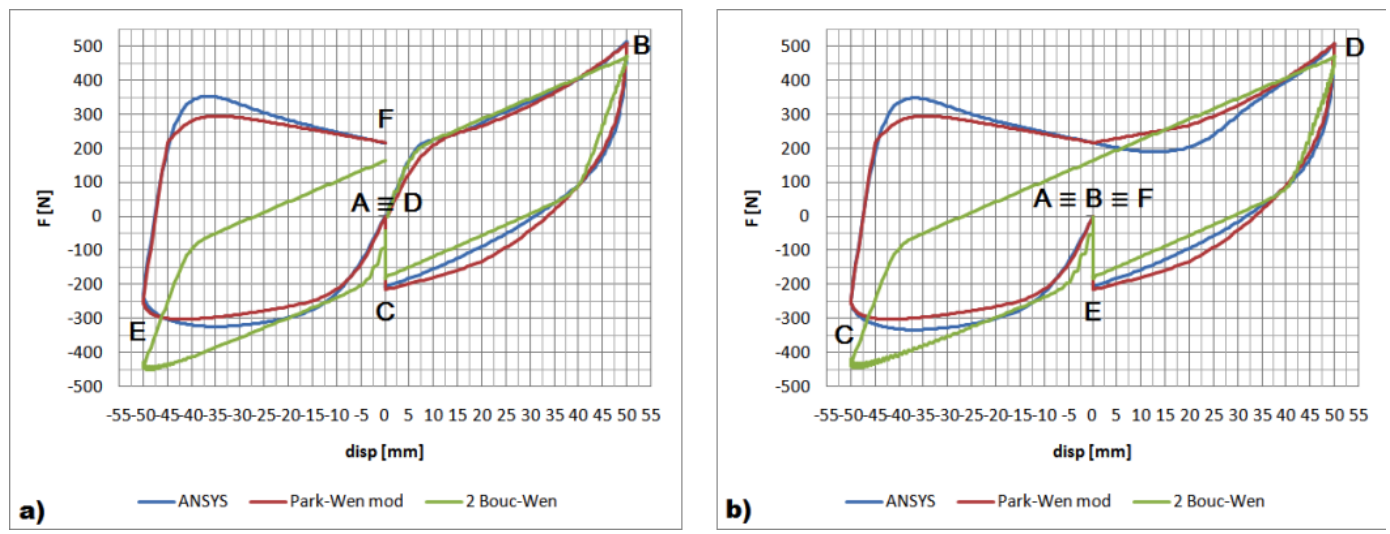

Figure 11: match between modified Park et al. model, ANSYS results and the previous device characterization (two independent Bouc-Wen models) for the scaled load path: (a) x-direction; (b) y-direction.

\section{Updated Bridge Benchmark Model and Seismic}

\section{Action}

The bridge under study is a is $1206 \mathrm{~m}$ long, fan-type cable stayed bridge (Figure 12), which crosses the Mississippi River near Cape Girardeau (USA), connecting Illinois and Missouri. The main span length is $350.6 \mathrm{~m}$. The deck is of mixed construction concrete-steel and is stiffened by longitudinal steel girders. 


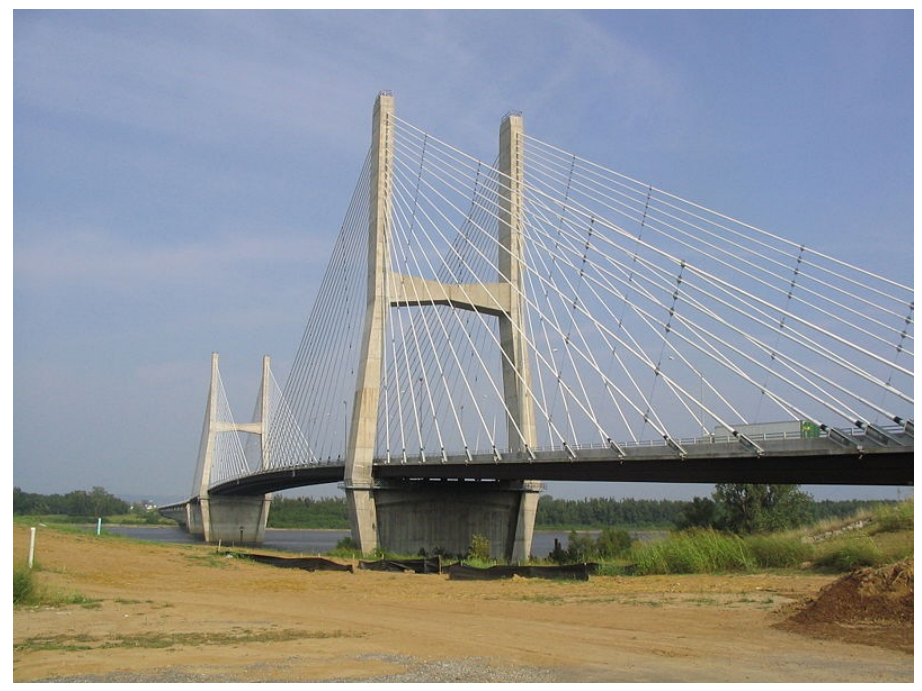

Figure 12: the Bill Emerson Memorial Bridge (Framerotblues, 2007; with permission)

One hundred and twenty eight stays of high-strength, low-relaxation, steel, arranged in a fantype configuration, support the deck. The smallest cable ha a cross-section area of $28.5 \mathrm{~cm}^{2}$ while the largest one has cross-section area of $76.3 \mathrm{~cm}^{2}$. The cable-stayed spans are supported by two towers while twelve additional piers support the Illinois approach spans. Each tower has a solid section below the cap beam, and a hollow section in the upper portion. For a more detailed description of the structure, as well as of its members, the reader is referred to [4].

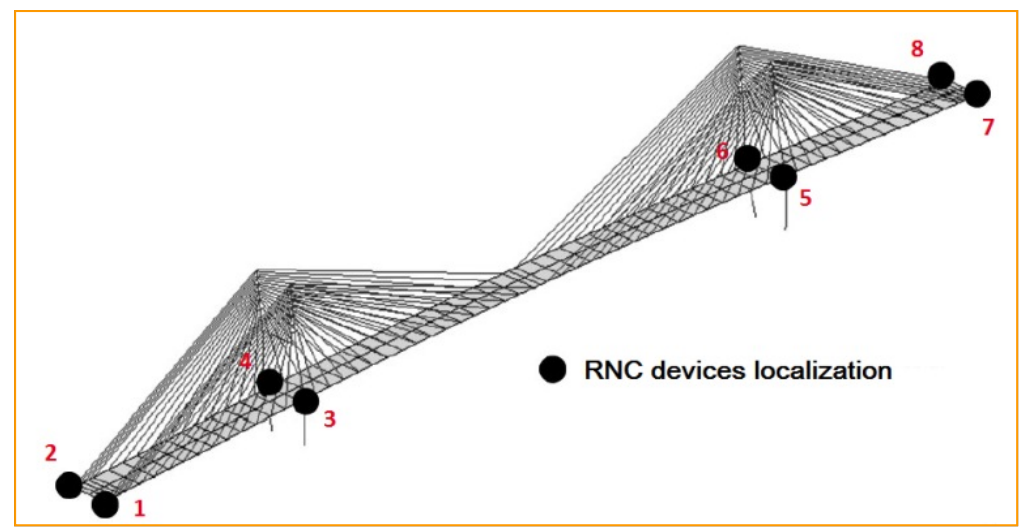

Figure 13: The Bill Emerson Memorial Bridge FEM model. The black dots mark the positions where RNC devices were added to the numerical model.

This bridge was the subject of a well-known benchmark on bridge control [4]. The model of the cable-stayed bridge adopted is set-up [2, 14, 15] in the ANSYS finite element framework enhancing the original benchmark model moving from a single rod type representation for each cable to a description with six rope elements per cable. This is to improve the modeling of the stays-deck coupled response. Furthermore, the non-linear response of the structure is also accounted for by evaluating the dynamic equilibrium of the structure in the deformed configuration at any instant of the simulations. Additionally, the numerical model [2, 17] comprises soil-structure interaction (in the vertical, transversal and longitudinal direction) through the use of impedance functions, lumped masses, springs and dampers at bents and piers.

The resulting finite element mesh in ANSYS comprises (Figure 13) linear beam elements for towers and the deck frame, linear shells elements for the concrete deck slab, tension only elements for the stay cables, totaling about 2600 nodes and 2800 elements. The materials are characterized as linear elastic. High performance concrete is adopted for the piers $\left(E=50 \times 106 \mathrm{kN} / \mathrm{m}^{2}\right)$; highstrength, low-relaxation steel for the stay cables $\left(E=210 \times 106 \mathrm{kN} / \mathrm{m}^{2}\right)$. The mixed structure of the deck (steel frame with concrete slab) is modeled by concrete shell elements connected to steel beams. The two materials retain the specified characteristics. A structural damping appropriate to 
this type of bridges (equal to 3\% of the critical one) is assigned to the bridge model as a Rayleigh type damping computed between the first $(0.28 \mathrm{~s})$ and the sixth $(0.64 \mathrm{~s})$ mode.

Soil-structure interaction is included in the bridge model. The ground motion is applied to the structure in the form of free-field ground displacements and velocities. These have been obtained from synthetic generated acceleration time histories, considering the spatial variability of the seismic ground motion. The acceleration time histories are obtained by the procedure presented in $[18,19]$, which relies on the spectral representation method by Shinozuka [20]. At the structuresoil contact points the auto-spectrum of the generated accelerations satisfy the well known KanaiTajimi Power Spectral Density (PSD) as modified by Clough and Penzien [21], while the crossspectra between the motions at different stations are derived from the coherency function proposed by Luco and Wong [22]. In the case at hand, a velocity of the shear waves $v_{s}=3000 \mathrm{~m} / \mathrm{s}$ and an incoherency factor $\alpha=0.2$ were adopted. Distinctively, the parameters of the Clough and Penzien PSD are chosen in order to minimize the difference between the value of the median response pseudo-acceleration spectrum with that prescribed by Eurocode 8 (EC8) [23]. Figure 14 depicts the average, over 10 realizations, pseudo-acceleration spectra with those imposed by EC8 for the horizontal and vertical component, respectively. The spectra are normalized to get a Peak Ground Acceleration of $1 \mathrm{~m} / \mathrm{s}^{2}$. 

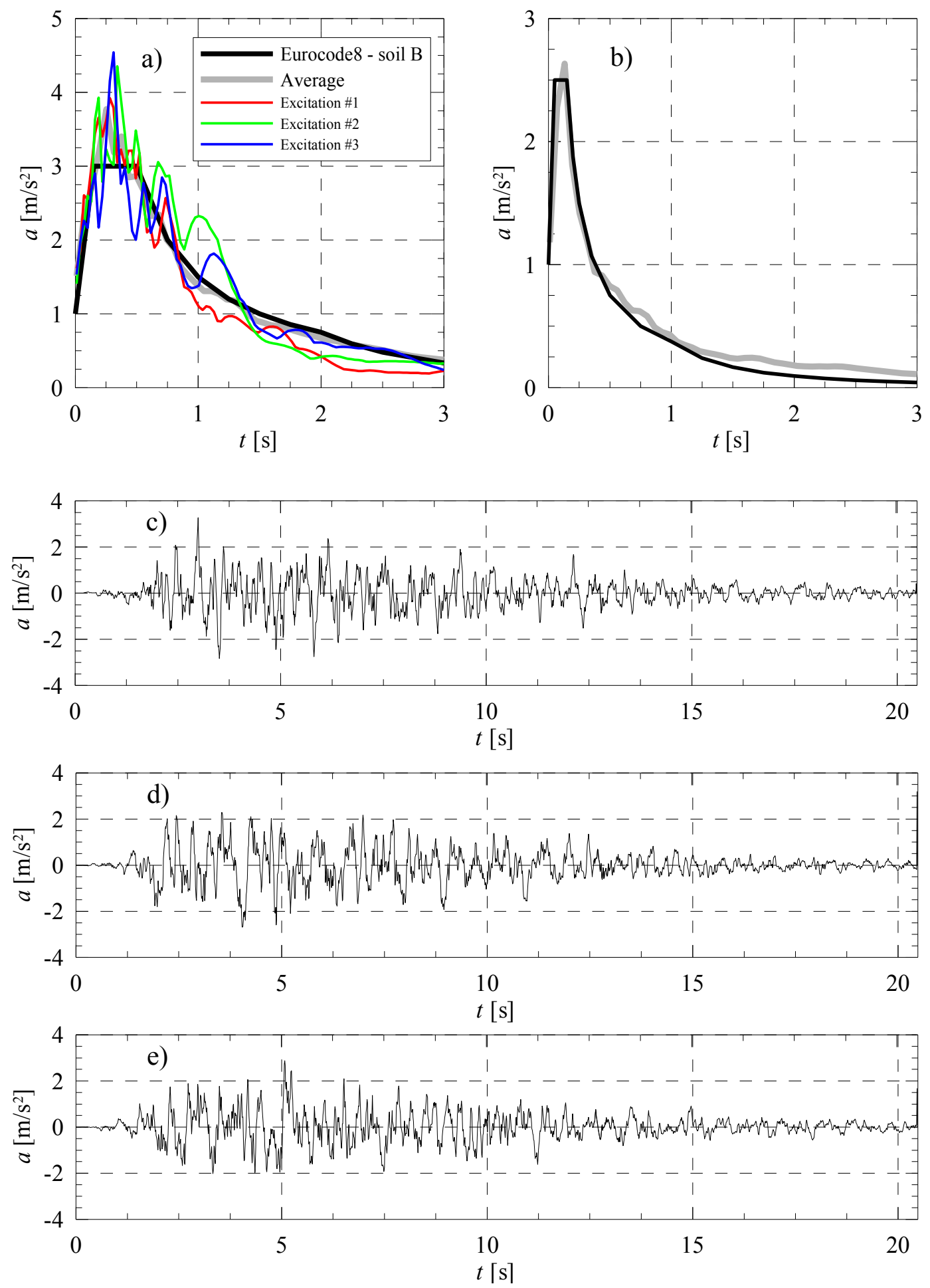

Figure 14: Average pseudo-acceleration spectra computed from 10 realizations of the ground motion for: a) horizontal component of ground acceleration, b) vertical component. Longitudinal acceleration at left bent for: c) Excitation \#1, d) Excitation \#2, e) Excitation \#3. 


\section{Seismic Performance of RNC on the Bridge}

\section{Structure}

Eight RNC devices are introduced in the bridge model, symmetrically with respect to the longitudinal axis, between deck and bents, and deck and piers at the locations highlighted by a black dot in Figure 13. The isolators correspond to the full-scale RNC device tested, since, referring to previous works [2], the parameters for this device are included in the optimal range of protection for the analyzed structure. The parameters for the reduced model are those in Section III-C.

The technique summarized in the previous section for generating the horizontal and vertical component of ground motion has been used to generate ten different excitations compatible with the horizontal response spectrum and 10 with the vertical response spectrum specified by EC 8 for soil type "B". Each time-history can be seen as a statistically independent realization of a stochastic process described by an appropriately modified Clough and Penzien [21] PSD to become spectrum-compatible. As such, two different realization belongs to a population that has the EC8 prescribed spectra as the average, they are completely uncorrelated and only share the time envelope function used to make them non-stationary in time (.a sample is shown if Figure $14 \mathrm{c}-\mathrm{d})$. The average acceleration response spectra, normalized at a PGA of $1 \mathrm{~m} / \mathrm{s}^{2}$, for the timehistories representing the horizontal ground motion and the vertical ground motions are depicted in Figure 14a and b, respectively. In Figure 14a the response spectra for the horizontal accelerations at the left bent in Excitations \#1 to \#3 are shown as well. Figure 14c-d depict the longitudinal, with respect to the bridge, acceleration at the left bent for the same excitations.

Six analyses were carried out in the time domain (Excitation \#1 to \#6), each one obtained by pairing two different seismic inputs in the horizontal direction and one in the vertical direction obtained as briefly described in the previous section. Comparisons between the two different bridge configurations (controlled and uncontrolled) will be shown in the following, in terms of average percentage reductions for the criteria listed in Table II.

Figures 15 and 16 summarize the outcomes of the numerical analyses on the bridge numerical model protected with RNC devices. The histograms in these figures represent the percentage reductions for the assumed performance criteria computed on the two different bridge configurations (controlled and uncontrolled).

\begin{tabular}{|l|l|}
\hline Criterion & \multicolumn{1}{c|}{ Response parameter } \\
\hline H1 & Maximum tower base bending moment in longitudinal direction \\
\hline H2 & Maximum tower base torque \\
\hline H3 & Maximum tower base bending moment in transversal direction \\
\hline H4 & Maximum tower base shear in longitudinal direction \\
\hline H5 & Maximum tower base shear in transversal direction \\
\hline H6 & Maximum mid-deck displacement in longitudinal direction \\
\hline H7 & Maximum mid-deck displacement in transversal direction \\
\hline H8 & Maximum longitudinal mid-deck acceleration \\
\hline H9 & Maximum transversal mid-deck acceleration \\
\hline
\end{tabular}

Table 2: Criteria for Assessing the Performance of the Isolation System 


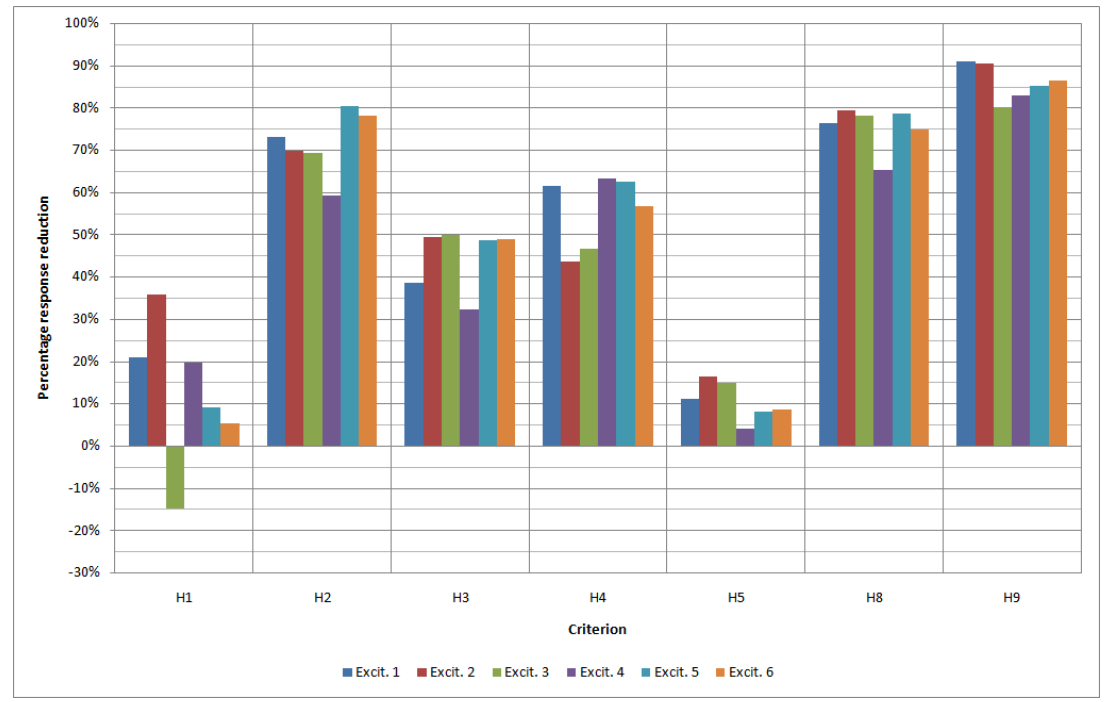

Figure 15: Percentage reductions for some of the assumed performance criteria in Table I computed on the controlled and uncontrolled bridge configurations.

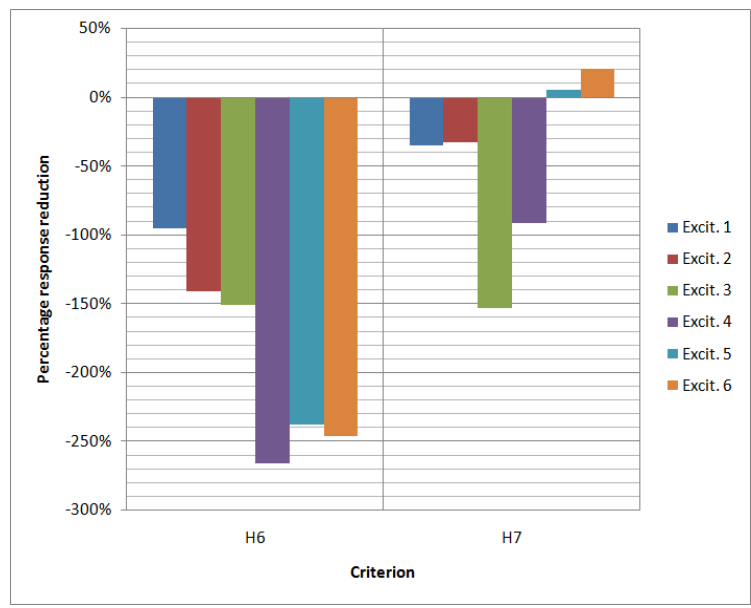

Figure 16: Percentage reductions for some of the assumed performance criteria in Table I computed on the controlled and uncontrolled bridge configurations.

Even if the buffer bidirectional formulation has to be confirmed by further physical tests, the mono-directional results and the consistent approach, with respect to the Park et al. model, justifies its use on the bridge model. Therefore, for Excitation \#6, Table 3 reports a coherent (same structural conditions) comparison in terms of relative difference for the criteria in Table 2 when the buffer mechanism in the RNC devices is excluded or included in the model. A small reduction of the longitudinal displacements (criterion H6) is reached when displacements are limited by the buffer mechanism of the RNC devices, with respect to the condition without buffer mechanism. This result is achieved at the cost of a small increment of the longitudinal acceleration of the deck (criterion H8). The remaining criteria do not show sensible variations.

Finally, aiming at a comparison with a previous study ([2]) on the bridge where the RNC devices were simulated by two orthogonal-unidirectional-uncoupled Bouc-Wen models, a comparison has been performed with the bridge model in [2] equipped with RNC devices simulated by the bidirectional model with buffer herein formulated. Table 4 lists, for seismic input $\# 4$ and \#5, the relative difference for the criteria listed in Table 2 coming from the two modeling approaches. It is worth underlining that the input \#4 is characterized by a strong bidirectional signature, much larger than for input \#5. One can observe from this comparison that a monodirectional modeling can not precisely follow the actual behavior of the isolator when this is affected by a non-negligible interaction in the two orthogonal direction. On the contrary, when the bidirectional effects are less important (input \#5) both approaches perform similarly. 


\begin{tabular}{|l|l|}
\hline Criterion & Relative difference [\%] \\
\hline H1 & 0.02 \\
\hline H2 & 4.99 \\
\hline H3 & 0.05 \\
\hline H4 & 0.00 \\
\hline H5 & -0.06 \\
\hline H6 & -6.00 \\
\hline H7 & 0.00 \\
\hline H8 & 3.89 \\
\hline H9 & -1.34 \\
\hline
\end{tabular}

Table 3: Comparison with and without buffer mechanism, input \#6.

For highlighting the differences between the two approaches in time domain, Figure 17 shows, for Excitation \#4, the time-history of the base shear in longitudinal (with respect to the deck development) direction at the base of the tower closer to the Illinois side.

\begin{tabular}{|c|c|c|}
\hline & Input \#4 & Input \#5 \\
\hline Criterion & $\begin{array}{c}\text { Relative difference } \\
{[\%]}\end{array}$ & $\begin{array}{c}\text { Relative difference } \\
{[\%]}\end{array}$ \\
\hline H1 & 19.70 & -0.53 \\
\hline H2 & -54.92 & -12.85 \\
\hline H3 & -8.65 & -1.82 \\
\hline H4 & -33.61 & -0.45 \\
\hline H5 & -5.19 & 0.26 \\
\hline H6 & -33.80 & -25.24 \\
\hline H7 & -105.92 & -24.95 \\
\hline H8 & 21.47 & -10.66 \\
\hline H9 & -310.00 & -7.71 \\
\hline
\end{tabular}

Table 4: Comparison between mono-directional and bi-directional formulation for the RNC device in the bridge model.

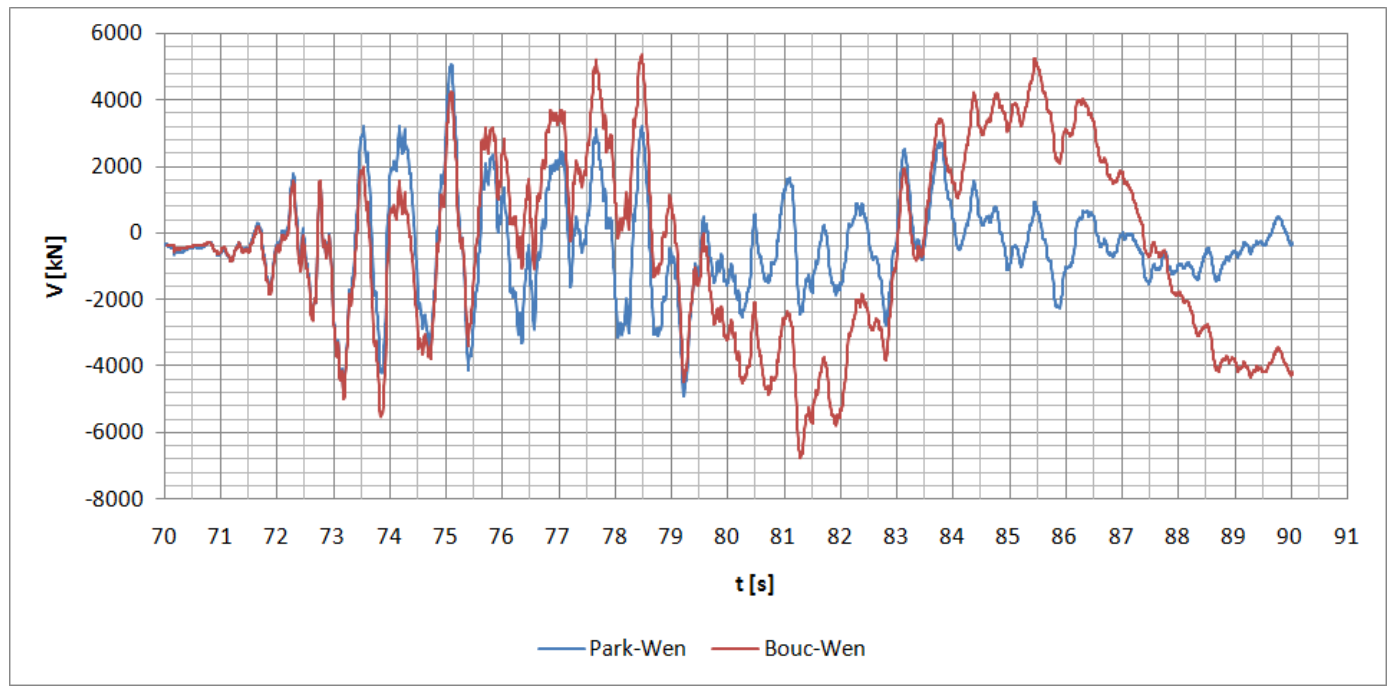


Figure 17: Base shear in longitudinal direction at one of the towers' bases, for Excitation \#4.

\section{Conclusions}

The laboratory tests and the analyses performed as part of this work offer an insight into the development of an innovative isolation device. The physical model of the RNC device successfully tested on the Exapod laboratory facility in unidirectional orthogonal loading paths, confirmed the specific characteristics of the dampers, as were identified by previous design studies. The laboratory tests allowed to confirm the preliminary design studies and to validate a new bidirectional model able to precisely follow the actual behavior of the isolator.

The laboratory tests and analyses performed lead to the following specific conclusions:

- The modified Park et al. model with optimal tuning of the parameters was proved able to match satisfactorily the physical behavior of the RNC. The same phenomenological model confirms to be effective also in reproducing the bidirectional response of the $3 \mathrm{D}$ finite element model of the dampers, including buckling of the same. This outcome will be very useful in planning and conducting further experiments on the physical model of the RNC isolator when bidirectional loading paths will be fully employed on the Hexapod facility.

- The RNCs devices, numerically simulated in the bridge analyses, were able to perform an effective reduction of the seismic effects both in terms of internal actions and accelerations. However, in agreement with the seismic isolation theory, this implied an increase of the displacements for the structure.

- The effect of the buffer mechanism on the bridge model slightly limits the peak displacements, at cost of a small increment of the deck accelerations. Internal forces did not show remarkable variations.

- A coupled bi-directional model (as the modified Park et al. one) is fundamental to precisely reproduce the actual behavior of the isolators when the deck motion is affected by a non-negligible interaction in the two (longitudinal-transversal) directions. Some performance criteria can vary by as much as $300 \%$ as a consequence.

The refined version of the ASCE bridge benchmark model confirms the positive contribution of the new dissipative isolation devices in the mitigation of the seismic effects in terms of internal actions and displacements of the structural components, as already emerged from previous studies carried out with a simpler model of the RNC device. Furthermore, the specific outcomes of the buffer mechanisms and the benefits coming from a full bidirectional formulation of the damper model have been assessed.

\section{References}

[1] Ismail, M. An innovative isolation device for aseismic design. PhD Thesis 2009; Doctoral program: Earthquake Engineering and Structural Dynamics, Technical University of Catalonia, Barcelona, Spain. (http://www.tdx.cat/handle/10803/6265), accessed 10 $0^{\text {th }}$ May 2014 20:08.

[2] Ismail, M., Rodellar, J., Carusone, G., Domaneschi, M., Martinelli, L. Characterization, modeling and assessment of roll-n-cage isolator using the cable stayed bridge benchmark. Acta Mechanica 2013; 224(3):525-547.

[3] Domaneschi, M., Martinelli, L., Shi, C. Aeolic and Seismic Structural Vibrations Mitigation on Long-Span CableSupported Bridges. Advanced Materials Research 2013; 690-693:1168-1171. doi:10.4028/www.scientific.net/AMR.690-693.1168.

[4] Caicedo, J.M., Dyke, S.J., Moon, S.J., Bergman, L.A., Turan, G., Hague,S. Phase II benchmark control problem for seismic response of cable-stayed bridges. Journal of Structural Control 2003; 10(3-4):137-168.

[5] ANSYS. Academic release 12 user manual, ANSYS, 2011.

[6] Ismail, M., Rodellar, J., Ikhouane, F. Method for the seismic isolation of a supported object. Patents No. WO2010000897A1, ES20080002043, P200802043, Spanish Office of Patents and Marks, 2008.

[7] Ismail, M., Casas, J.R., Rodellar, J. Near-fault isolation of cable-stayed bridges using RNC isolator. Engineering Structures 2013; 56:327-342.

[8] Ismail, M., Rodellar, J. Experimental mechanical characterization of a rolling-based seismic isolation system. Proc. 6WCSCM, Sixth World Conference on Structural Control and Monitoring 2014; Barcelona, Spain.

[9] Ismail, M., Rodellar, J. A mechanical extension for Stewart platform (Hexapod). Patent No. P201331348, Spanish Office of Patents and Marks, 2013.

[10] Ismail, M., Rodellar, J. Experimental mechanical characterization of a rolling-based seismic isolation system. Proc. 6WCSCM, Sixth World Conference on Structural Control and Monitoring 2014; Barcelona, Spain. 
[11] De Mari, G. Controllo strutturale di ponti strallati mediante sistema RNC - Formulazione bidimensionale del sistema di controllo (in Italian) 2014. MSc Thesis A.Y. 2013-2014; Politecnico di Milano, Milan I.

[12] Park, Y.J., Wen, Y.K., Ang, K.S.. Random vibration of hysteretic systems underbi-directional ground motions. Earthquake Engineering and Structural Dynamics 1986; 14:543-557.

[13] Wen, Y. Method for random vibration of hysteretic systems. Journal of the Engineering Mechanics Division ASCE 1976; 102(2):246-263.

[14] MATLAB. Release R2013a user manual, MATLAB, 2013.

[15] Domaneschi, M., Martinelli, L. Performance Comparison of Passive Control Schemes for the Numerically Improved ASCE Cable-Stayed Bridge Model. Earthquakes and Structures 2012; 3(2):181-201. ISSN: 2092-7614 (Print), ISSN: 2092-7622 (Online).

[16] Domaneschi, M., Martinelli, L.. Extending the Benchmark Cable-Stayed Bridge for Transverse Response under Seismic Loading. Journal of Bridge Engineering ASCE 2014; 19(3). DOI: 10.1061/(ASCE)BE.1943-5592.0000532.

[17] Domaneschi, M., Limongelli, M.P., Martinelli, L. Vibration Based Damage Localization Using MEMS on a Suspension Bridge Model. Smart Structures and Systems 2013; 12(6):679-694.

[18] Martinelli, L., Barbella, G., Feriani, A. Modeling of Qiandao Lake submerged floating tunnel subject to multi-support seismic input. Procedia Engineering 2010; 4:311-318.

[19] Martinelli, L., Barbella, G., Feriani, A. A numerical procedure for simulating the multi-support seismic response of submerged floating tunnels anchored by cables. Eng Struct 2011;33:2850-2860.

[20] Shinozuka, M. Monte Carlo solution of structural dynamics. Comput Struct 1972; vol 2(5-6):855-874.

[21] Clough, R.W., Penzien, J. Dynamics of structures 1975; New York, NY, McGraw-Hill.

[22] Luco, J.E., Wong, H.L. Response of a rigid foundation to a spatially random ground motion. Earthq Eng Struct Dyn $1986 ; 14: 891-908$

[23] Eurocode 8 EN 1998-1. Design of structures for earthquake resistance 2005. Part 1: General rules, seismic actions and rules for buildings. 